\title{
FOXK1 promotes glioblastoma proliferation and metastasis through activation of Snail transcription
}

\author{
HAITAO XU, SHULAN HUANG, XIAONAN ZHU, WANGCHENG ZHANG and XIANGYANG ZHANG \\ Department of Neurosurgery, Renmin Hospital of Wuhan University, Wuhan, Hubei 430060, P.R. China
}

Received June 27, 2016; Accepted September 9, 2017

DOI: $10.3892 /$ etm.2018.5732

\begin{abstract}
Forkhead box K1 (FOXK1) has been identified to have a crucial function in development and oncogenesis. However, its role in glioblastoma has remained largely elusive and was therefore assessed in the present study. In human glioblastoma multiforme (GBM) tissue samples, FOXK1 was determined to be highly expressed compared with adjacent normal tissue samples. In addition, high levels of FOXK1 were detected in the T98G and LN18 GBM cell lines as compare with those in normal human astrocytes. Of note, high expression of FOXK1 was revealed to be associated with metastasis and tumor size. Loss- and gain-of-function experiments were then performed to determine whether FOXK1 regulates epithelial to mesenchymal transition (EMT) and cell proliferation. Knockdown of FOXK1 significantly suppressed EMT and metastasis of GBM cells, while ectopic expression of FOXK1 promoted them. A luciferase reporter assay and a chromatin immunoprecipitation assay revealed that FOXK1 activated the transcription of Snail. In addition, as the results indicated that FOXK1 promotes GBM cell proliferation, the potential effect of FOXK1 on the cell cycle and apoptosis were further assessed. While FOXK1 had no effect on apoptosis, it promoted cell proliferation via enhancing the S-phase population. In brief, the present study indicated that FOXK1 acts as an oncogene with a key function in glioblastoma cell proliferation and EMT.
\end{abstract}

\section{Introduction}

Of all human brain tumors, $>80 \%$ are gliomas, which have a poor prognosis with a 5-year survival rate of $<5 \%$ (1). Glioblastoma multiforme (GBM) accounts for $15 \%$ of brain tumors (2). Although multiple methods are available to treat glioblastoma multiforme (GBM), including radiotherapy,

Correspondence to: Dr Haitao Xu, Department of Neurosurgery, Renmin Hospital of Wuhan University, 238 Jiefang Road, Wuhan, Hubei 430060, P.R. China

E-mail: haitaoxu2016@sina.com

Key words: forkhead box K1, epithelial to mesenchymal transition, snail, metastasis, cell cycle surgery, chemotherapy and photodynamic therapy $(3,4)$, survival of patients remains poor. Therefore, it is necessary to understand the pathogenic processes at the molecular level in order to identify novel markers and molecular targets that may improve the diagnosis, predict outcomes and provide novel treatment approaches.

The forkhead box (FOX) family of proteins are involved in multiple crucial biological processes and human diseases $(5,6)$. FOXK1 was first reported in 1994 as a DNA-binding protein, which specifically binds to the CCAC box motif (7). FOXK1 contains a forkhead domain and a forkhead-associated domain, which bear a DNA-binding region and a phosphopeptide recognition region, respectively $(8,9)$. FOXK1 has been identified to interact with four and a half LIM domains 2 in myogenic progenitor cells (10). In addition, FOXK1 interacts with Sin3 protein via the Sin3-interacting domain, thereby regulating myogenic progenitors (10). FOXK1 also takes part in development and oncogenesis (11). However, the role of FOXK1 in GBM has remained elusive.

Epithelial to mesenchymal transition (EMT) is a complex process, during which cells lose their epithelial properties while gaining mesenchymal characteristics. Multiple epithelial and mesenchymal markers are known, including E-cadherin as an epithelial marker and $\mathrm{N}$-cadherin as a mesenchymal marker. E-cadherin is an important protein that participates in cell anchoring junctions, so that EMT results in loss of cell-to-cell contact and therefore facilitates cell motility; therefore, EMT promotes tumor cell metastasis $(12,13)$. Several studies indicated that multiple transcription factors are highly expressed during EMT, including Snail, Slug, ZEB and TWIST, which repress E-cadherin transcription (14-16).

In order to explore the molecular mechanisms of the effects of FOXK1 in GBM, the present study detected FOXK1 expression levels in GBM tissues by reverse transcription-quantitative polymerase chain reaction (RT-qPCR) and revealed that FOXK1 was not only highly expressed, but positively associated with tumor size and metastasis. In addition, it was demonstrated that FOXK1 facilitates EMT through activation of the transcription of Snail. Furthermore, fluorescence-assisted cell sorting (FACS) analysis indicated that FOXK1 promotes GBM cell proliferation through regulating the cell cycle. In brief, FOXK1, as a crucial transcription factor, has a key function in GBM cell proliferation and EMT. 


\section{Materials and methods}

GBM tissue samples and cell lines. A total of 83 pairs of GBM tumor tissues and adjacent non-tumorous tissues were collected from the neurosurgery department of Renmin Hospital of Wuhan University between 2013 and 2016. All GBM patients were histologically confirmed. Clinical data, including patient age, gender, tumor size and metastasis were collected from the information system of Renmin Hospital of Wuhan University. All patients have provided informed consent for use of their data/specimens. All tissue experiments were approved by the Ethics Committee of Wuhan University (Wuhan, China).

The T98G and LN18 human GBM cell lines, and normal human astrocytes (NHAs) derived from XCL-1 GFAPp-Nanoluc-Halotag (ATCC ${ }^{\circledR}$ ACS $-5006^{\mathrm{TM}}$ ) were purchased from the American Type Tissue Collection (Manassas, VA, USA) and cultured in Dulbecco's modified Eagle's medium (DMEM; HyClone, Logan, UT, USA) containing $10 \%$ fetal bovine serum (FBS; HyClone) at $37^{\circ} \mathrm{C}$ a humidified atmosphere containing $5 \% \mathrm{CO}_{2}$.

Western blot analysis. To obtain total protein, cells were lysed with radioimmunoprecipitation assay buffer containing protease inhibitor cocktail (Sigma-Aldrich; Merck KGaA, Darmstadt, Germany). Following centrifugation at $13,000 \mathrm{x} \mathrm{g}$ for $10 \mathrm{~min}$ at $4^{\circ} \mathrm{C}$, the protein concentration in the supernatant was determined using a bicinchoninic protein assay kit (Pierce; Thermo Fisher Scientific, Inc., Waltham, MA, USA). Total protein (50 $\mu \mathrm{g}$ per lane) was subjected to $10 \%$ SDS-PAGE and transferred protein onto nitrocellulose membranes (EMD Millipore, Billerica, MA, USA). Membranes were then blocked in $5 \%$ non-fat milk at room temperature for $1 \mathrm{~h}$, followed by incubation with primary antibodies at $4^{\circ} \mathrm{C}$ overnight. Following washing in PBS containing Tween 20, membranes were then incubated with horseradish peroxidase-secondary antibodies (1:5,000; Abcam, Cambridge, MA, USA; cat. nos. ab6721 and ab97023) at room temperature for $1 \mathrm{~h}$. Finally, protein bands were visualized by enhanced chemiluminescence (Thermo Fisher Scientific, Waltham, MA, USA; cat. no. 32106). The following antibodies were used: FOXK1 (1:2,000; Abcam; cat. no. ab172730), EMT antibody kit (1:2,000; Cell Signaling Technology, Inc., Danvers, MA, USA; cat. no. 9782) and $\beta$-actin (1:5,000; Sigma-Aldrich; Merck KGaA, Darmstadt, Germany; cat. no. A2228). $\beta$-actin served as an internal control.

Cell transfection. T98G and LN18 cells were cultured to 60-70\% confluence and transfected with PCMV-Tag2B vector, FOXK1 (Vigene Biosciences, Inc., Rockville, MD, USA), scramble RNA (SCR) or FOXK1 siRNA (Sigma-Aldrich; Merck KGaA) using the Lipofectamine 2000 reagent (Thermo Fisher Scientific, Inc., Waltham, MA, USA; cat. no. 11668019) according to the manufacturer's protocol. Following $48 \mathrm{~h}$ of transfection, cells were used to subsequent experiments.

Anchorage-independent cell growth assay. Following knockdown or ectopic expression FOXK1 in T98G and LN18 cells (transfection time, $48 \mathrm{~h}$ ), $\sim 4 \times 10^{4}$ cells were re-suspended with $2 \mathrm{ml}$ DMEM supplemented with $0.35 \%$ agarose and layered onto $2 \mathrm{ml}$ of $0.6 \%$ agarose/medium in the 6-well plates. Cells were cultured with $2 \mathrm{ml}$ fresh growth medium every 3 days for 15 days. Colonies were stained with $0.5 \%$ crystal violet and counted. $n>50$ were defined as a colony. All experiments were performed at least three times.

RT-qPCR.TRIzolreagent(Invitrogen; ThermoFisherScientific, Inc.) was used to extract total RNA from GBM/adjacent tissue samples or cells according to the manufacturer's instructions. Complementary (c)DNA was then generated using TransScript First-Strand cDNA Synthesis SuperMix (TransGen, Beijing, China). SYBR MIX (Roche Diagnostics, Basel, Switzerland) was used to perform PCR in the 7500 Real-Time PCR System to detect relative mRNA expression. Primers were designed as follows: E-cadherin forward, 5'-AAACATCATTGATGC AGACC-3' and reverse, 5'-GATAGATTCTTGGGTTGG GTC-3'; $\alpha$-catenin forward, 5'-TGTTACACAGGTTACAAC CCT-3' and reverse, 5'-GATCATCTGCGAACTCTCCT-3'; $\mathrm{N}$-cadherin forward, 5'-CAAAGCCTGGAACATATGTG-3'; and reverse, 5'-GTTTGAAAGGCCATATGTGG-3'; fibronectin forward, 5'-AATGTGAACGACACATTCCA-3' and reverse, 5'-ACCACTTGAGCTTGGATAGG-3'; Snail forward, 5'-TCTAATCCAGAGTTTACCTTCC-3'; and reverse, 5'-GAA GAGACTGAAGTAGAGGAG-3'; Slug forward, 5'-ACACAT ACAGTGATTATTTCCC-3'; and reverse, 5'-GAGGAGGTG TCAGATGGA-3'; TWIST forward, 5'-CCAGGTACATCG ACTTCCTC-3'; and reverse, 5'-GGAAACAATGACATC TAGGTCTC-3'; FOXK1 forward, 5'-CAGTTACCGCTTTGT GCAG-3'; and reverse, 5'-GAATTCTGCCAGCCTTTGTC-3'; GAPDH forward, 5'-ATTTCCTGGTATGACAACGA-3' and reverse, 5'-TTGATGGTACATGACAAGGTG-3'. GAPDH was used as an internal control. The following thermocycling conditions were used: $5 \mathrm{~min}$ at $98^{\circ} \mathrm{C}$, denaturation at $98^{\circ} \mathrm{C}$ for $30 \mathrm{sec}$, annealing at $57^{\circ} \mathrm{C}$ for $30 \mathrm{sec}$ and extension at $72^{\circ} \mathrm{C}$ for $40 \mathrm{sec}$, performed for 30 cycles. The relative expression of gene was analyzed using the $2^{-\Delta \Delta C q}$ method (17). All experiments were performed at least three times.

Transwell migration assay. LN18 cells with knockdown or ectopic expression of FOXK1 (transfection time, $48 \mathrm{~h}$ ) were seeded onto Transwell membrane inserts (Corning, Inc., Corning, NY, USA) at $5 \times 10^{3}$ cells per well in DMEM without serum. DMEM containing 10\% FBS was added to the lower chamber. Following incubation for $8 \mathrm{~h}$ at $37^{\circ} \mathrm{C}$, cells were fixed in $4 \%$ paraformaldehyde, followed by staining with $0.5 \%$ crystal violet at room temperature for $15 \mathrm{~min}$. For each membrane, migrated cells in 6 random fields at x20 magnification were counted. All experiments were performed in triplicate.

Cell Counting kit (CCK)-8 assay. Cell proliferation was detected using a CCK-8 assay as previously described (18). In brief, T98G and LN18 cells were transfected with empty vector or FLAG-FOXK1, or with control small interfering RNA (siRNA) or FOXK1 siRNA for $48 \mathrm{~h}$ and then seeded in 96-well plates at $4 \times 10^{3}$ cells/well in $200 \mu 1$ DMEM containing $10 \%$ FBS. Following incubation for $24 \mathrm{~h}, 20 \mu \mathrm{l} \mathrm{CCK}-8$ stain in $200 \mu \mathrm{l}$ DMEM was added to each well, followed by incubation for $2 \mathrm{~h}$ at $37^{\circ} \mathrm{C}$. Finally, the absorbance of each well was measured at $450 \mathrm{~nm}$. 
Table I. Clinicopathological parameters of patients with glioblastoma multiforme $(\mathrm{n}=83)$.

\begin{tabular}{|c|c|c|c|c|c|}
\hline \multirow[b]{2}{*}{ Parameter } & \multirow[b]{2}{*}{$\mathrm{n}(\%)$} & \multicolumn{2}{|c|}{ FOXK1 protein expression } & \multirow[b]{2}{*}{ P-value } & \multirow[b]{2}{*}{ Chi-square values } \\
\hline & & Low $(\mathrm{n}=30) \%$ & High $(n=53) \%$ & & \\
\hline \multicolumn{6}{|l|}{ Gender } \\
\hline Male & $51(61.4)$ & $18(21.6)$ & $33(39.8)$ & 0.839 & 0.041 \\
\hline Female & $32(38.6)$ & $12(14.5)$ & $20(24.1)$ & & \\
\hline \multicolumn{6}{|c|}{ Age (years) } \\
\hline$\geq 40$ & $49(59.0)$ & $21(25.4)$ & $28(33.7)$ & 0.126 & 2.335 \\
\hline$<40$ & $34(41.0)$ & $9(10.8)$ & $25(30.1)$ & & \\
\hline \multicolumn{6}{|c|}{ Tumor size $(\mathrm{cm})$} \\
\hline$\geq 2$ & $43(51.8)$ & $5(6)$ & $38(45.8)$ & $<0.001$ & 23.236 \\
\hline$<2$ & $40(48.2)$ & $25(30.1)$ & $15(18.1)$ & & \\
\hline \multicolumn{6}{|c|}{ Pathological grade } \\
\hline I-II & $39(47.0)$ & $19(22.9)$ & $20(24.1)$ & 0.025 & 5.039 \\
\hline III-IV & $44(53.0)$ & $11(13.2)$ & $33(39.8)$ & & \\
\hline \multicolumn{6}{|l|}{ Metastasis } \\
\hline Yes & $44(53.0)$ & $7(8.4)$ & $37(44.6)$ & $<0.001$ & 16.613 \\
\hline No & $39(47.0)$ & $23(27.7)$ & $16(19.3)$ & & \\
\hline
\end{tabular}

FOXK1, forkhead box K1.

Apoptosis and cell cycle analysis. For apoptosis and cell cycle analysis, T98G and LN18 cells with knockdown or ectopic expression of FOXK1 (transfection time, $48 \mathrm{~h}$ ) were collected and washed three times with PBS. Following fixation in $70 \%$ cold ethanol, cells were stained with Annexin V-propidium iodide (PI) solution or PI solution for apoptosis or cell cycle analysis, respectively. Finally, FACS analysis was performed to detect apoptosis and cell cycle distribution. All experiments were performed at least three times.

Chromatin immunoprecipitation (ChIP) assay. The ChIP assay was performed using an EZ ChIP Kit (EMD Millipore). In brief, cells were lysed and sonicated to produce chromatin fragments of $200-1 \times 10^{3} \mathrm{bp}$. Immunoprecipitated chromatin was detected using $3 \mu \mathrm{g}$ anti-FOXK1 antibody (ab18196; Abcam, Cambridge, MA, USA) and specific primers were used to amplify the target gene promotor region. The same quantity of Immunoglobulin G (IgG; ab172730; Abcam) served as a negative control. The PCR assay was performed using 2X EasyTaq PCR SuperMix (TransGene) according to the manufacturer's instructions. The PCR conditions were as follows: $5 \mathrm{~min}$ at $98^{\circ} \mathrm{C}$, denaturation at $98^{\circ} \mathrm{C}$ for $30 \mathrm{sec}$, annealing at $57^{\circ} \mathrm{C}$ for $30 \mathrm{sec}$ and extension at $72^{\circ} \mathrm{C}$ for $40 \mathrm{sec}$, performed for 30 cycles. The primers used were as follows: TWIST forward, 5'-AGGCGCTATCAAATTCCC-3' and reverse, 5'-AAGGCAGCAGAGCCAGAG-3'; Snail forward, 5'-ATGGCAGCTCACTGTGGC-3' and reverse, 5'-CGCTGG CTTCCTTTCATT-3'; Slug forward, 5'-CCACCTCACCCT CCAAAC-3' and reverse, 5'-CACATGAAGATCACCCTA-3'. The products were detected by agarose gel electrophoresis with ethidium bromide staining. Input groups served as a positive control and $\mathrm{IgG}$ groups served as a negative control.
Luciferase reporter assay. Cells were seeded in 6-well plates at $6 \times 10^{4}$ cells/well and transfected with the vector, FOXK1, pGL3-Snail, Renilla (Vigene Biosciences, Inc.) as the cell density reached $70-80 \%$ confluence. After $24 \mathrm{~h}$ of transfection, an illuminometer was used to quantify the luciferase activity. All experiments were performed at least three times.

Statistical analysis. All results were analyzed with SPSS 21.0 statistical software (IBM Corp., Armonk, NY, USA). The association between FOXK1 expression and clinicopathological parameters was evaluated by Pearson's Chi-square test. All values are expressed as the mean \pm standard error of the mean. The expression of FOXK1 in GBM cell lines and NHAs was analyzed with the Chi-squared test. The two-tailed Student's t-test was used to assess differences between two groups. The survival analysis was performed using Kaplan-Meir analysis. Analysis of variance followed by Tukey's post hoc test was used to assess the differences between multiple groups. $\mathrm{P}<0.05$ was considered to indicate a statistically significant difference.

\section{Results}

FOXK1 is overexpressed in GBM tissues and cell lines. FOXK1 is a crucial transcription factor, and several studies regarded it as a tumor suppressor, while multiple studies also indicated that FOXK1 promotes EMT and cell metastasis. The role of FOXK1 in GBM has remained to be elucidated. The present study first examined the expression of FOXK1 in human GBM tissues and adjacent non-tumorous tissues using RT-qPCR. In a total of 83 pairs of human GBM and adjacent non-tumorous tissue samples, FOXK1 was overexpressed in 


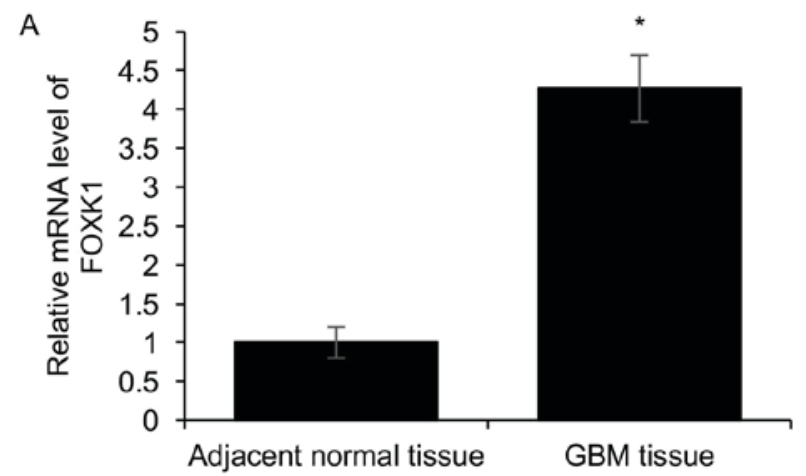

B

NHA LN18 T98G
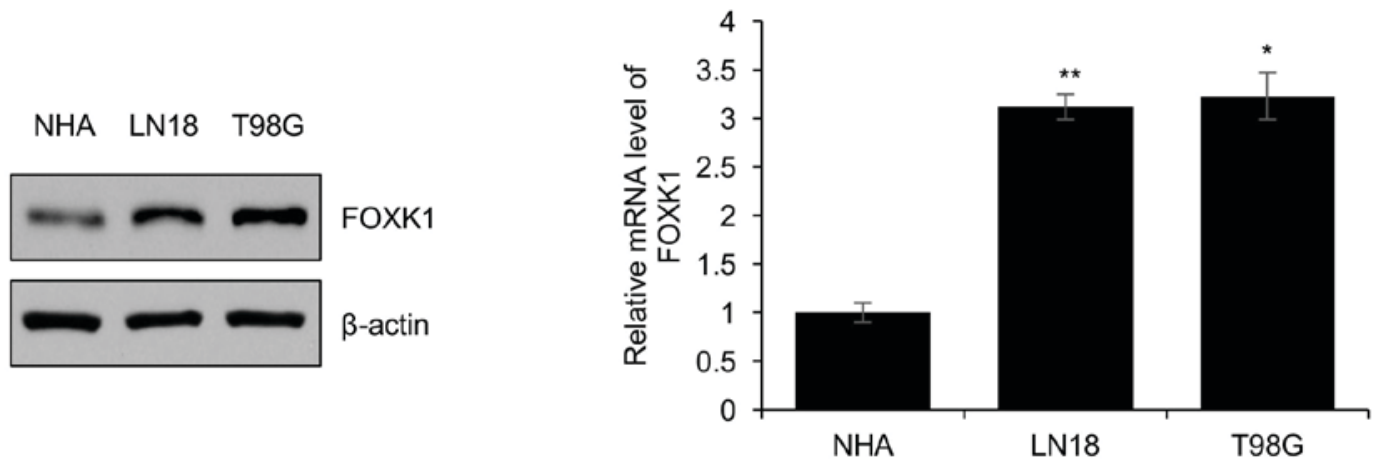

C

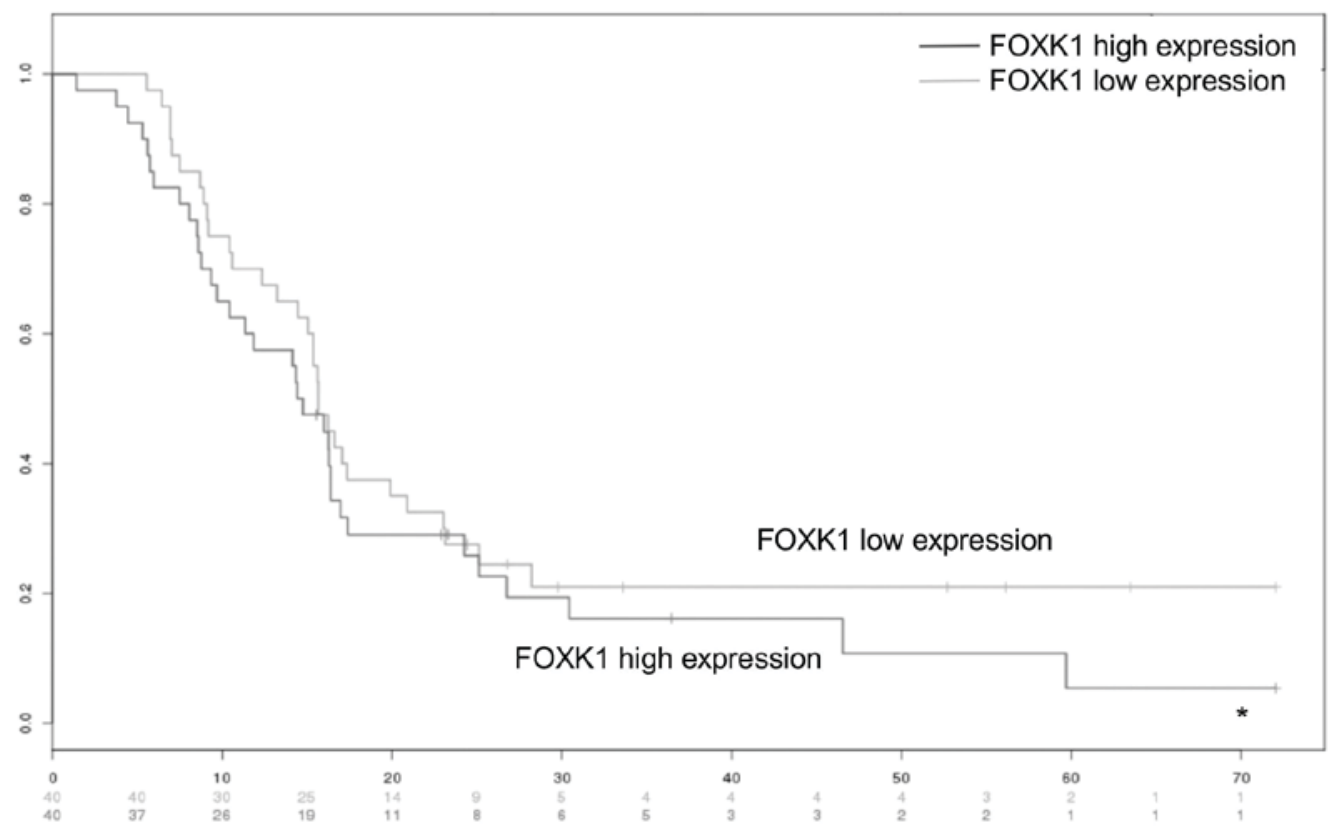

Figure 1. FOXK1 is highly expressed in glioblastoma tissue and cells. (A) RT-qPCR analysis was used to analyze FOXK1 expression in 83 pairs of GBM tumors and adjacent non-tumorous tissue samples. (B) FOXK1 protein and mRNA expression in glioblastoma cell lines and NHAs by western blotting and RT-qPCR, respectively. ${ }^{*} \mathrm{P}<0.05,{ }^{* *} \mathrm{P}<0.01$ vs. non-tumorous samples or NHAs. (C) Kaplan-Meier survival estimates indicating the prognostic significance of FOXK1 expression in GBM patients. "P<0.05 vs. FOXK1 low expression. RT-qPCR, reverse transcription-quantitative polymerase chain reaction; FOXK1, forkhead box K1; GBM, glioblastoma multiforme; NHAs, normal human astrocytes.

the GBM tissues compared with that in adjacent non-tumorous tissues (Fig. 1A).

In addition, the expression of FOXK1 was identified to be positively associated with metastasis, tumor size and tumor stage. However, the age and gender of the patients were not associated with FOXK1 (Table I). Furthermore, the expression of FOXK1 was detected in the T98G and LN18 GBM cell lines as well as in NHAs. The results indicated that, compared with that in NHAs, FOXK1 was obviously overexpressed in the
GBM cell lines (Fig. 1B). Consequently, the influence of FOXK1 expression on the survival of GBM patients was analyzed. The results demonstrated that the 5-year survival in the group with low FOXK1 expression was significantly higher than that in the group with high expression of FOXK1 (Fig. 1C).

FOXK1 promotes EMT of GBM cells through activation of SNAIL transcription. EMT promotes the cancer cell metastasis ability of most carcinomas. High expression of FOXK1 
A
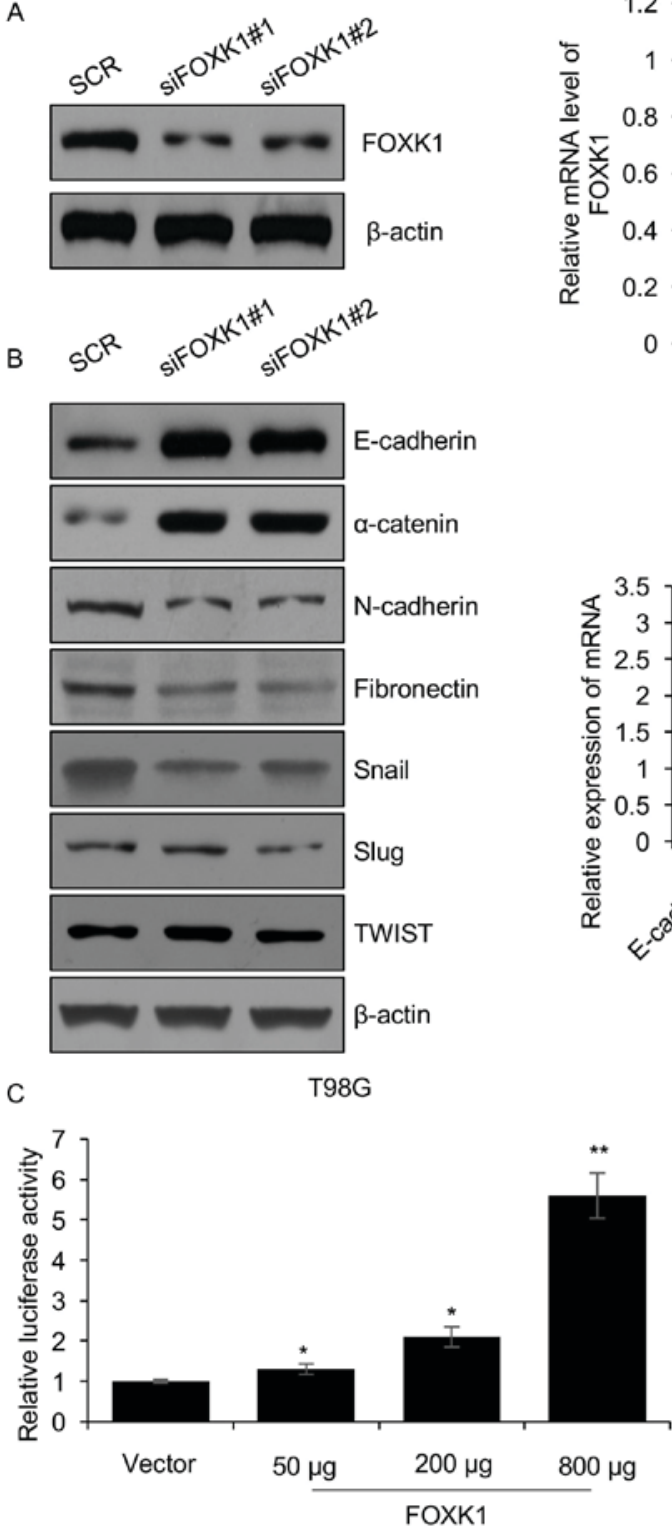
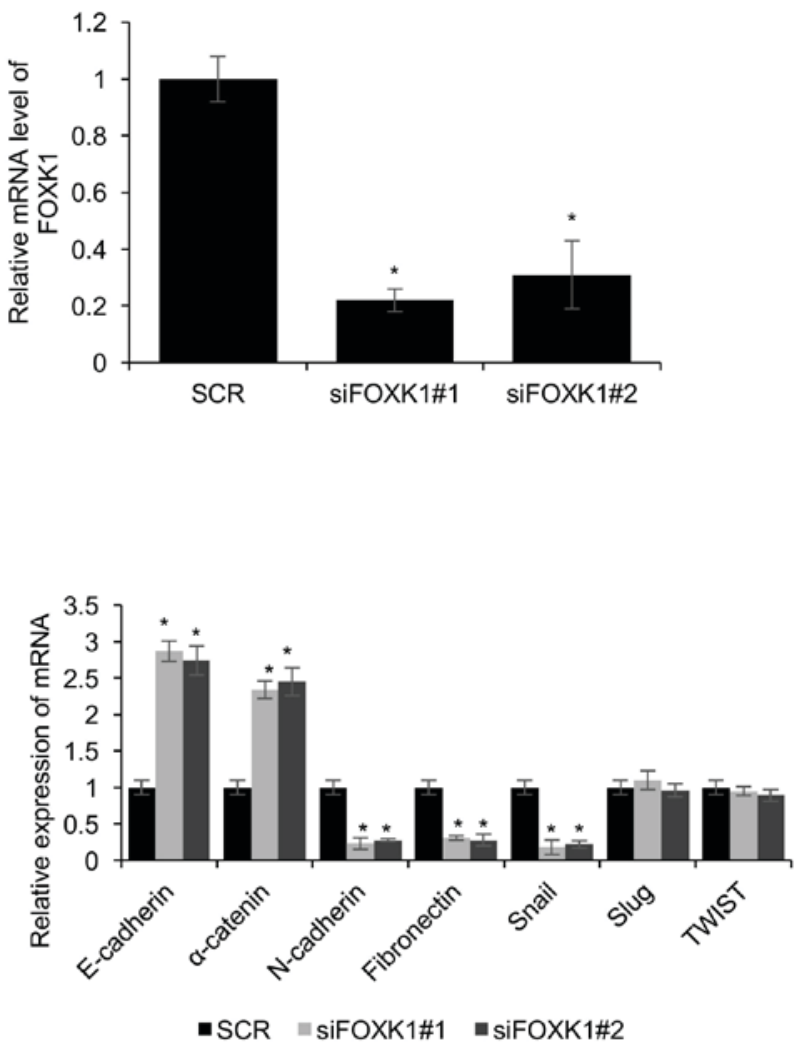

D

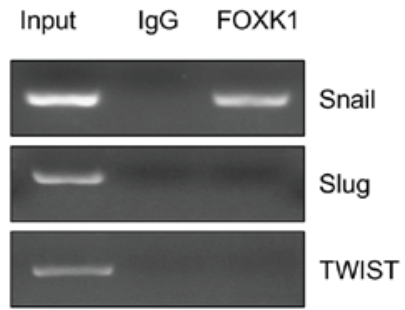

Figure 2. FOXK1 promotes EMT through transcriptional activation of SNAIL in glioblastoma multiforme cells. (A) Two different siRNAs were utilized to knockdown FOXK1 in T98G cells. After transfection for 48 h, FOXK1 knockdown efficiency was assessed by western blotting and RT-qPCR. (B) Knockdown of FOXK1 regulates EMT-associated protein expression. FOXK1 depletion led to suppression of the mesenchymal marker N-cadherin and fibronectin, but promoted the expression of the epithelial markers E-cadherin and $\alpha$-catenin. The protein and mRNA levels were detected by western blotting and RT-qPCR, respectively. (C) Co-transfection with SNAIL-Luciferase reporter vector and Renilla construct, and different amounts of FOXK1 expression vector (50, 200 or $800 \mathrm{ng} /$ well) in T98G and LN18 cells. After transfection for $36 \mathrm{~h}$, cells were collected and subjected to the luciferase reporter assay. All experiments were performed at least three times. ${ }^{*} \mathrm{P}<0.05,{ }^{* *} \mathrm{P}<0.01 \mathrm{vs}$. SCR/control group. (D) A chromatin immunoprecipitation assay was performed in T98G cells with rabbit normal IgG or anti-FOXK1, followed by PCR with specific primers for SNAIL. PCR products were indicated. EMT, epithelial to mesenchymal transition; PCR, polymerase chain reaction; FOXK1, forkhead box K1; IgG, immunoglobulin G; SCR, scrambled control; siFOXK1, small interfering RNA targeting FOXK1.

is closely associated with metastasis, which suggested that FOXK1 may participate in the EMT. To test this hypothesis, FOXK1 was knocked down by FOXK1 siRNA in T98G cells and the knockdown efficiency was assessed. As presented in Fig. 2A, FOXK1 was depleted by $70-80 \%$. Furthermore, EMT markers were also significantly changed. At the mRNA 
A

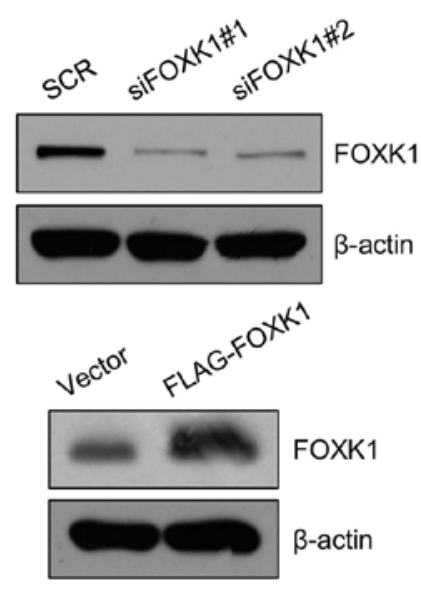

B

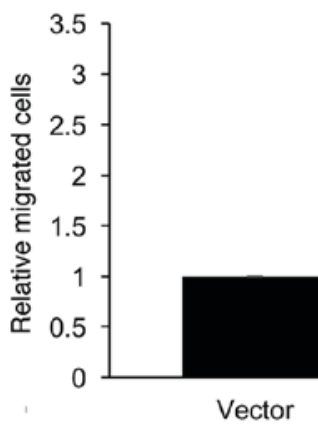

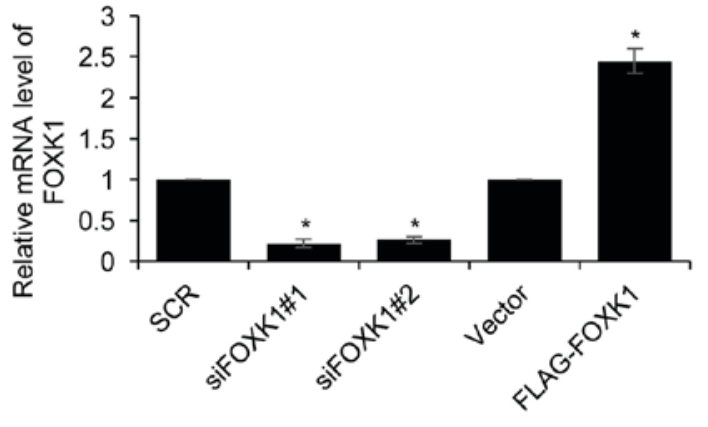

C

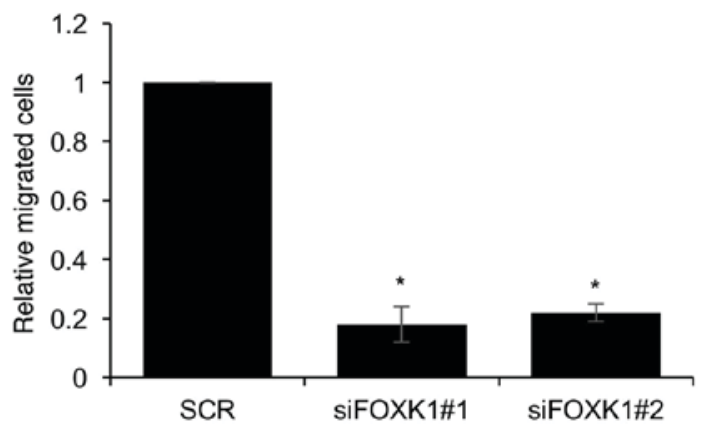

D

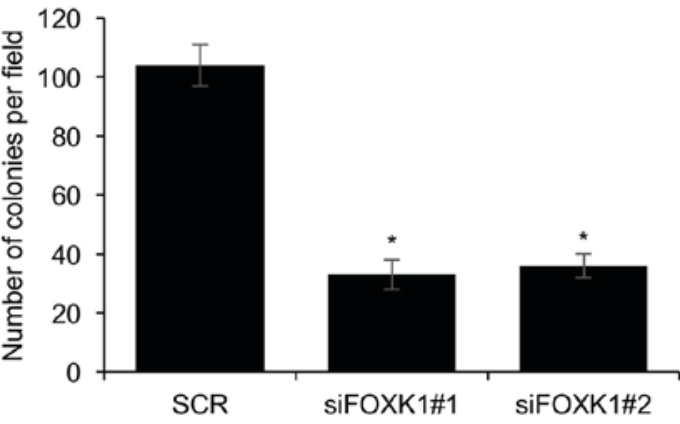

Figure 3. FOXK1 facilitates glioblastoma cell metastasis. (A) Following knockdown or ectopic expression of FOXK1 in LN18 cells, the protein or mRNA levels of FOXK1 were detected by western blotting or reverse transcription-quantitative polymerase chain reaction, respectively. (B and C) Transwell migration assay of LN18 cells after (B) ectopic expression and (C) knockdown of FOXK1 (48 h post-transfection). (D) Anchorage-independent cell growth assay. LN18 cells were transfected with FOXK1 siRNA and then placed on soft agar; the average number of colonies formed was determined. All experiments were performed at least three times. "P<0.05 vs. SCR/empty vector group. FOXK1, forkhead box K1; SCR, scrambled control; siFOXK1, small interfering RNA targeting FOXK1.

as well as at the protein level, the expression of the epithelial markers E-cadherin and $\alpha$-catenin was increased, while that of the mesenchymal markers $\mathrm{N}$-cadherin and fibronectin was obviously decreased (Fig. 2B). Furthermore, the expression of Snail, a transcription factor that promotes EMT, was decreased when FOXK1 was silenced, whereas Slug and TWIST were not affected (Fig. 2B). As Snail is an important transcription factor which promotes the EMT process, it was then further explored whether FOXK1 promotes EMT through transcriptional regulation of SNAIL. For this purpose, a luciferase reporter assay was utilized to determine the effect of FOXK1 on SNAIL promoter activity. As presented in Fig. 2C, FOXK1 transcriptionally activated the SNAIL gene promoter in T98G and LN18 cells. In addition, the ChIP assay demonstrated that endogenous FOXK1 bound to the promoter region of SNAIL, but not Slug and TWIST (Fig. 2D). In brief, the present results revealed that FOXK1 activated the transcription of SNAIL.

FOXK1 facilitates GBM cell metastasis. An enhanced capacity to form metastasis is a characteristic of cancer that is associated with the EMT, and metastasis is the reason for poor prognosis. As the above mentioned results suggested that FOXK1 promotes EMT through transcriptional activation of SNAIL, it was hypothesized that FOXK1 regulates GBM cell metastasis. In order to verify this hypothesis, a Transwell migration assay was performed using LN18 cells in which FOXK1 was ectopically overexpressed or knocked down as verified by western blot analysis and RT-qPCR (Fig. 3A). The Transwell assay revealed that overexpression 
A

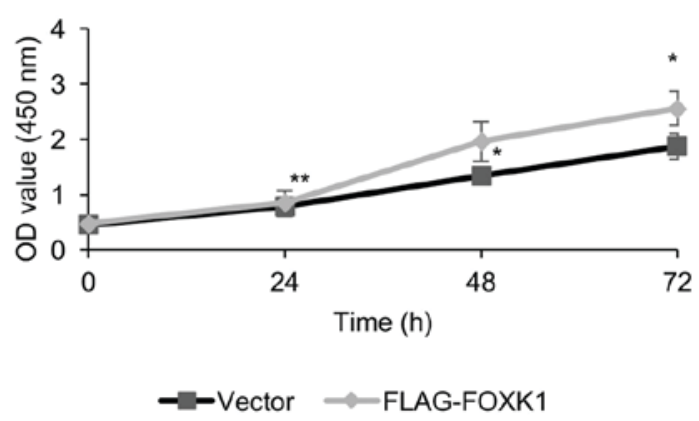

C

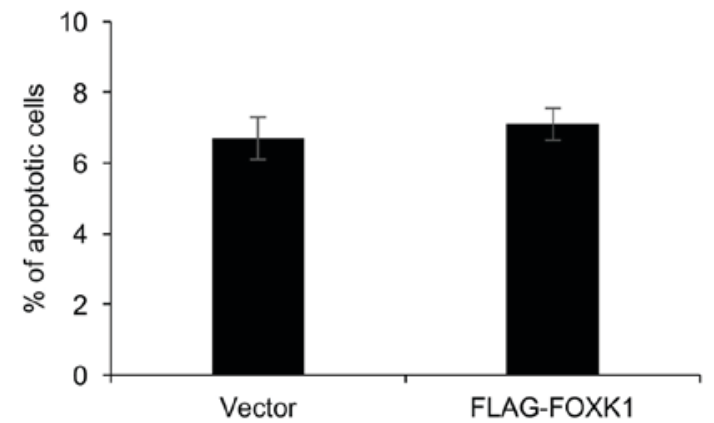

E
B

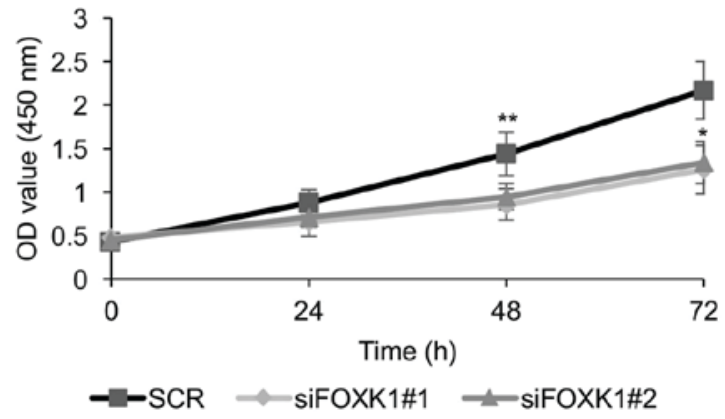

D
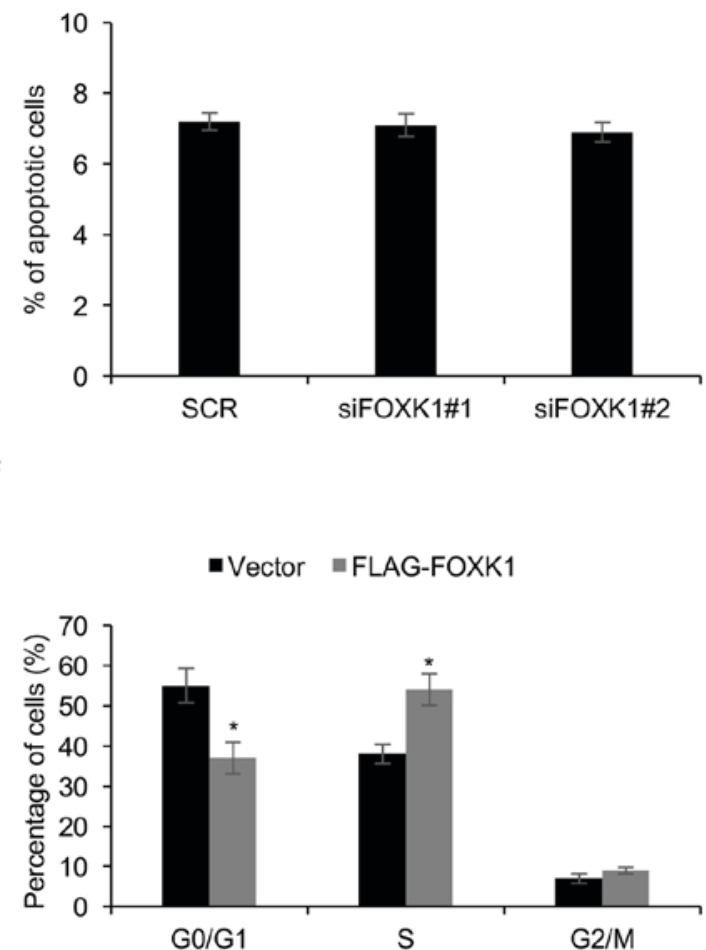

Figure 4. FOXK1 expression changes glioblastoma cell proliferation through regulating the cell cycle. (A and B) Cell Counting kit-8 assays were performed to assess the effect of (A) ectopic expression or (B) knockdown of FOXK1 on the viability of T98G cells. ${ }^{*} \mathrm{P}<0.05,{ }^{* *} \mathrm{P}<0.01$ vs. SCR/empty vector group. (C and D) Cell apoptosis assays were performed to assess the effect of (C) ectopic expression or (D) knockdown of FOXK1 on cell apoptosis of T98G cells ${ }^{*} \mathrm{P}<0.05,{ }^{* *} \mathrm{P}<0.01$ vs. SCR/empty vector group. (E and F) Flow cytometric analysis of the cell cycle of T98G cells following (E) FOXK1 knockdown and (F) ectopic expression of FOXK1. "P $<0.05$ vs. SCR/empty vector group. Percentages of cell cycle populations are displayed in the bar graph. FOXK1, forkhead box K1; SCR, scrambled control; siFOXK1, small interfering RNA targeting FOXK1; OD, optical density.

of FOXK1 in LN18 cells increased the number of cells that transgressed through the membrane compared with that in the control group (Fig. 3B). The opposite result was seen when FOXK1 was silenced in LN18 cells, as the number of cells transgressed to the lower side of the membrane was significantly decreased (Fig. 3C). In addition, the anchorage-independent cell growth assay demonstrated that silencing of FOXK1 expression suppressed colony formation (Fig. 3D). All of these results supported that FOXK1 stimulated cell migration.

FOXK1 levels affect GBM cell proliferation through regulating the cell cycle. Considering the higher expression of FOXK1 in GBM tumor tissues than in adjacent non-tumorous tissues and high expression of FOXK1 being positively associated with the tumor size, it was speculated that FOXK1 may regulate GBM cell proliferation. In order to determine the role of FOXK1 in cell proliferation, T98G cells with ectopic expression of FOXK1 or knockdown were subjected to a CCK8 assay. The results demonstrated that FOXK1 overexpression obviously increased T98G cell growth, whereas FOXK1 knockdown inhibited T98G cell growth (Fig. 4A and B).

To further explore the mechanism by which FOXK1 enhances cell proliferation, it was first assessed whether FOXK1 suppressed cell apoptosis, but no marked effect of FOXK1 overexpression or knockdown on the apoptotic rates of T98G cells was observed (Fig. 4C and D). Next, the effect of FOXK1 on the cell cycle was assessed using FACS. The results 
indicated that following knockdown of FOXK1 in T98G cells, the percentage of cells in the $G_{0} / G_{1}$ phase was obviously increased, while the percentage of cells in the S-phase was significantly decreased (Fig. 4E). By contrast, overexpression of FOXK1 in T98G cells decreased the percentage of cells in the $G_{0} / G_{1}$ phase and obviously increased the percentage of cells in the S-phase (Fig. 4F). A similar result was seen in LN18 cells (data not shown). The above results revealed that FOXK1 expression promoted GBM cell proliferation through regulating the cell cycle.

\section{Discussion}

Gliomas account for almost $80 \%$ of brain tumors and GBM has become the most prevalent subtype of gliomas $(19,20)$. It is difficult to treat malignant gliomas, and the survival of GBM patients under treatment is only 12-15 months $(21,22)$.

The members of the forkhead family have multiple functional roles during embryogenesis (23-25). Several studies indicated that FOX proteins have crucial roles in various cancer types $(26,27)$. For instance, FOXA1 has been reported to be highly expressed in numerous cancer types, including bladder (28), breast (29), prostate (30) and pancreatic cancers (31). The roles of FOXK1, as a member of the FOX family, have remained to be fully elucidated in cancer, and it has been reported to have tumor suppressor (32) as well as oncogenic funcions (33).

The present study reported that FOXK1 is highly expressed in GBM tumor tissues compared with that in adjacent normal tissues. Analysis of GBM cell lines compared with NHAs provided similar results. As expected, high expression of FOXK1 was positively associated with several clinicopathological characteristics, namely tumor size and metastasis. These results indicated that FOXK1 has a crucial function in GBM development.

Metastasis is the major cause of cancer-associated mortality, causing $>90 \%$ of fatalities of carcinoma patients, and EMT promotes the metastasis ability of cancer cells for most carcinomas. Multiple reasons have been suggested for the low incidence of extracranial metastasis of GBM, including inhibition of extracranial growth of glioblastoma cells through the immune system (34), the blood-brain barrier (35), short survival periods (36) or the absence of lymphatic channels in the central nervous system (37), and a previous report indicated extracranial metastases after organ transplantation from GBM donors (38). In the present study, it was demonstrated that the transcription factor FOXK1 takes part in regulating the EMT. Knockdown of endogenous FOXK1 was sufficient to suppress EMT. This observation was consisted with previous study (33). The present study indicated that FOXK1 regulated Snail, a key transcription factor which promotes EMT. The luciferase reporter assay and the ChIP assay confirmed that FOXK1 activates Snail transcription. In addition, the Transwell assay and the anchorage-independent cell growth assay indicated that FOXK1 promotes GBM cell metastasis.

The CCK-8 and colony formation assay revealed that ectopic expression of FOXK1 significantly promoted GBM cell proliferation, whereas depletion of FOXK1 suppressed cell proliferation. As previous reports have demonstrated, FOXK1 promotes cell proliferation in prostate cancer (39), colorectal cancer (40) and gastric cancer (41). In order to investigate whether the enhancing effect of FOXK1 on cell proliferation is caused by inhibition of apoptosis or promotion of the cell cycle, cell apoptosis was assessed after FOXK1 was silenced; however, no significant effect was observed. Next, the cell cycle assay demonstrated that ectopic expression of FOXK1 caused an obvious increase in the percentage of cells in the S-phase and decreased the percentage of cells in the $\mathrm{G}_{0} / \mathrm{G}_{1}$-phase. These results inferred that FOXK1 promotes cell proliferation through facilitating cell cycle progression, but the detailed molecular mechanisms remain to be elucidated by further studies.

\section{References}

1. Dunbar E and Yachnis AT: Glioma diagnosis: Immunohistochemistry and beyond. Adv Anat Pathol 17: 187-201, 2010.

2. Young RM, Jamshidi A, Davis G and Sherman JH: Current trends in the surgical management and treatment of adult glioblastoma. Ann Transl Med 3: 121, 2015.

3. Stylli SS, Howes M, MacGregor L, Rajendra P and Kaye AH: Photodynamic therapy of brain tumours: Evaluation of porphyrin uptake versus clinical outcome. J Clin Neurosci 11: 584-596, 2004.

4. Stylli SS, Kaye AH, MacGregor L, Howes M and Rajendra P: Photodynamic therapy of high grade glioma-long term survival. J Clin Neurosci 12: 389-398, 2005.

5. Hannenhalli S and Kaestner KH: The evolution of Fox genes and their role in development and disease. Nat Rev Genet 10: 233-240, 2009.

6. Jackson BC, Carpenter C, Nebert DW and Vasiliou V: Update of human and mouse forkhead box (FOX) gene families. Hum Genomics 4: 345-352, 2010

7. Bassel-Duby R, Hernandez MD, Yang Q, Rochelle JM, Seldin MF and Williams RS: Myocyte nuclear factor, a novel winged-helix transcription factor under both developmental and neural regulation in striated myocytes. Mol Cell Biol 14: 4596-4605, 1994.

8. Clark KL, Halay ED, Lai E and Burley SK: Co-crystal structure of the HNF-3/fork head DNA-recognition motif resembles histone H5. Nature 364: 412-420, 1993.

9. Durocher D and Jackson SP: The FHA domain. FEBS Lett 513: 58-66, 2002.

10. Shi X, Bowlin KM and Garry DJ: Fhl2 interacts with Foxk1 and corepresses Foxo4 activity in myogenic progenitors. Stem Cells 28: 462-469, 2010.

11. Carlsson P and Mahlapuu M: Forkhead transcription factors: Key players in development and metabolism. Dev Biol 250: 1-23, 2002.

12. Thiery JP: Epithelial-mesenchymal transitions in tumour progression. Nat Rev Cancer 2: 442-454, 2002.

13. Bailey JM, Singh PK and Hollingsworth MA: Cancer metastasis facilitated by developmental pathways: Sonic hedgehog, Notch, and bone morphogenic proteins. J Cell Biochem 102: 829-839, 2007.

14. Nieto MA, Sargent MG, Wilkinson DG and Cooke J: Control of cell behavior during vertebrate development by Slug, a zinc finger gene. Science 264: 835-839, 1994.

15. Cano A, Pérez-Moreno MA, Rodrigo I, Locascio A, Blanco MJ, del Barrio MG, Portillo F and Nieto MA: The transcription factor snail controls epithelial-mesenchymal transitions by repressing E-cadherin expression. Nat Cell Biol 2: 76-83, 2000.

16. Yang J, Mani SA, Donaher JL, Ramaswamy S, Itzykson RA, Come C, Savagner P, Gitelman I, Richardson A and Weinberg RA: Twist, a master regulator of morphogenesis, plays an essential role in tumor metastasis. Cell 117: 927-939, 2004.

17. Livak KJ and Schmittgen TD: Analysis of relative gene expression data using real-time quantitative PCR and the 2(-Delta Delta C(T)) method. Methods 25: 402-408, 2001.

18. Cohen MS, Hussain HB and Moley JF: Inhibition of medullary thyroid carcinoma cell proliferation and RET phosphorylation by tyrosine kinase inhibitors. Surgery 132: 960-967, 2002. 
19. Zhu Y and Parada LF: The molecular and genetic basis of neurological tumours. Nat Rev Cancer 2: 616-626, 2002.

20. Schwartzbaum JA, Fisher JL, Aldape KD and Wrensch M: Epidemiology and molecular pathology of glioma. Nat Clin Pract Neurol 2: 494-503, 2006.

21. Stupp R, Hegi ME, Mason WP, van den Bent MJ, Taphoorn MJ, Janzer RC, Ludwin SK, Allgeier A, Fisher B, Belanger K, et al: Effects of radiotherapy with concomitant and adjuvant temozolomide versus radiotherapy alone on survival in glioblastoma in a randomised phase III study: 5-year analysis of the EORTC-NCIC trial. Lancet Oncol 10: 459-466, 2009.

22. Cloughesy TF, Cavenee WK and Mischel PS: Glioblastoma: From molecular pathology to targeted treatment. Annu Rev Pathol 9: 1-25, 2014

23. Katoh $M$ and Katoh M: Human FOX gene family (Review). Int J Oncol 25: 1495-1500, 2004

24. Yang Y, Hou H, Haller EM, Nicosia SV and Bai W: Suppression of FOXO1 activity by FHL2 through SIRT1-mediated deacetylation. EMBO J 24: 1021-1032, 2005.

25. Murakami H, Aiba H, Nakanishi M and Murakami-Tonami Y: Regulation of yeast forkhead transcription factors and FoxM1 by cyclin-dependent and polo-like kinases. Cell Cycle 9: 3233-3242, 2010.

26. Teh MT, Wong ST, Neill GW, Ghali LR, Philpott MP and Quinn AG: FOXM1 is a downstream target of Glil in basal cell carcinomas. Cancer Res 62: 4773-4780, 2002.

27. Müller SM, Terszowski G, Blum C, Haller C, Anquez V, Kuschert S, Carmeliet P, Augustin HG and Rodewald HR: Gene targeting of VEGF-A in thymus epithelium disrupts thymus blood vessel architecture. Proc Natl Acad Sci USA 102: 10587-10592, 2005.

28. Reddy OL, Cates JM, Gellert LL, Crist HS, Yang Z, Yamashita H, Taylor JA III, Smith JA Jr, Chang SS Cookson MS, et al: Loss of FOXA1 drives sexually dimorphic changes in urothelial differentiation and is an independent predictor of poor prognosis in bladder cancer. Am J Pathol 185: 1385-1395, 2015.

29. Badve S, Turbin D, Thorat MA, Morimiya A, Nielsen TO, Perou CM, Dunn S, Huntsman DG and Nakshatri H: FOXA1 expression in breast cancer-correlation with luminal subtype A and survival. Clin Cancer Res 13: 4415-4421, 2007.

30. Mirosevich J, Gao N, Gupta A, Shappell SB, Jove R and Matusik RJ: Expression and role of Foxa proteins in prostate cancer. Prostate 66: 1013-1028, 2006.
31. Jungert K, Buck A, von Wichert G, Adler G, König A, Buchholz M, Gress TM and Ellenrieder V: Sp1 is required for transforming growth factor-beta-induced mesenchymal transition and migration in pancreatic cancer cells. Cancer Res 67: 1563-1570, 2007.

32. Sun T, Wang H, Li Q, Qian Z and Shen C: Forkhead box protein $\mathrm{k} 1$ recruits TET1 to act as a tumor suppressor and is associated with MRI detection. Jpn J Clin Oncol 46: 209-221, 2016.

33. Wu Y, Peng Y, Wu M, Zhang W, Zhang M, Xie R, Zhang P, Bai Y, Zhao J, Li A, et al: Oncogene FOXK1 enhances invasion of colorectal carcinoma by inducing epithelial-mesenchymal transition. Oncotarget 7: 51150-51162, 2016.

34. Fonkem E, Lun M and Wong ET: Rare phenomenon of extracranial metastasis of glioblastoma. J Clin Oncol 29: 4594-4595, 2011

35. Liwnicz BH and Rubinstein LJ: The pathways of extraneural spread in metastasizing gliomas: A report of three cases and critical review of the literature. Hum Pathol 10: 453-467, 1979.

36. Stupp R, Mason WP, van den Bent MJ, Weller M, Fisher B, Taphoorn MJ, Belanger K, Brandes AA, Marosi C, Bogdahn U, et al: Radiotherapy plus concomitant and adjuvant temozolomide for glioblastoma. N Engl J Med 352: 987-996, 2005.

37. Pasquier B, Pasquier D, Lachard A, N'Golet A, Panh MH and Couderc P: Extraneural metastasis of central nervous system tumours (author's transl). Bull Cancer 66: 25-28, 1979 (In French).

38. Müller C, Holtschmidt J, Auer M, Heitzer E, Lamszus K, Schulte A, Matschke J, Langer-Freitag S, Gasch C, Stoupiec M, et al: Hematogenous dissemination of glioblastoma multiforme. Sci Transl Med 6: 247ra101, 2014.

39. Chen F, Xiong W, Dou K and Ran Q: Knockdown of FOXK1 suppresses proliferation, migration and invasion in prostate cancer cells. Oncol Res 25: 1261-1267, 2017.

40. Wu M, Wang J, Tang W, Zhan X, Li Y, Peng Y, Huang X, Bai Y, Zhao J, Li A, et al: FOXK1 interaction with FHL2 promotes proliferation, invasion and metastasis in colorectal cancer. Oncogenesis 5: e271, 2016.

41. Peng Y, Zhang P, Huang X, Yan Q, Wu M, Xie R, Wu Y, Zhang M, Nan Q, Zhao J, et al: Direct regulation of FOXK1 by $\mathrm{C}$-jun promotes proliferation, invasion and metastasis in gastric cancer cells. Cell Death Dis 7: e2480, 2016. 